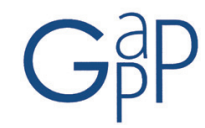

GESTIÓN Y ANÁLISIS DE POLÍTICAS PÚBLICAS, Nueva Época, nº 12 julio-diciembre 2014 ISSN: 1989-8991

DOI: http://dx.doi.org/10.24965/gapp.voi12.10206

\title{
Una aproximación a la eficiencia técnica de las empresas de transporte urbano colectivo en España
}

\author{
Pedro Manuel Balboa La Chica \\ pbalboa@defc.ulpgc.es \\ Margarita Mesa Mendoza \\ mmesa@defc.ulpgc.es \\ Heriberto Suárez Falcón \\ heriberto.suarez@ulpgc.es
}

Recibido: 13 de octubre de 2014

Aceptado: 20 noviembre 2014

\section{Resumen}

En el presente trabajo se analiza la eficiencia técnica de las empresas de transporte urbano colectivo en España durante el período 2008 -2011. El estudio se realiza tanto a nivel global como segmentado según el tamaño de las empresas, utilizando una metodología no paramétrica (DEA). $A$ tal fin, partiendo de la información económico-financiera de estas empresas, se seleccionan los inputs y outputs para aplicar un modelo orientado a inputs con rendimientos variables a escala. Los resultados evidencian que pocas empresas se sitúan en la frontera de eficiencia, y que las más eficientes no son sólo las empresas de mayor tamaño.

Palabras Clave

Empresas de transporte urbano, eficiencia, costes, finanzas.

\section{An Approach to the Technical Efficiency of Urban Transportation Companies in Spain}

\section{Abstract}

In this paper the technical efficiency of urban public transport companies in Spain during the period 2008-2011 is analyzed. The study is conducted globally and also segmented by size of business, using a nonparametric approach (DEA). To this end, based on the financial information of these companies the inputs and outputs are selected to apply an input oriented variable returns to scale model. The results show that few companies are at the efficient frontier, and that the most efficient firms are not only larger.

\section{Keywords}

Urban transport companies, efficiency, finance, cost. 


\section{INTRODUCCIÓN}

Es de destacar que todo proceso de ordenación del territorio necesita de una adecuada planificación de los transportes que dirija debidamente los flujos de tráfico. Separar los planes de urbanismo de los de transporte conduce al colapso en la circulación y de ello tenemos múltiples ejemplos.

En consecuencia, los problemas del tráfico responden a la inadaptación de las vías existentes a las necesidades de circulación, que se agrava por la coexistencia del transporte individual y colectivo. Así, por ejemplo, el tráfico en zonas urbanas es causa de confrontación entre intereses particulares y sociales.

Tal como reconoce Thomson (1976: 13), el transporte "nos lleva a donde necesitamos ir, pero no siempre por la ruta más lógica”. Así, el objetivo del economista se basa fundamentalmente en el conocimiento y cuantificación de los recursos utilizados, tratando de que satisfagan, en la mayor medida posible, las necesidades humanas, persiguiendo en muchas ocasiones un beneficio social más que un beneficio económico.

A título indicativo, y según datos del Instituto Nacional de Estadística, durante el año 2013 el transporte urbano en España fue utilizado por más de 2.700 millones de viajeros. No obstante, autores como De Rus y Herce (1996: 20) sostienen que la importancia del transporte en la economía va más allá de las variables macroeconómicas. Precisamente, por cuestiones como ésta y tal como indica López del Pino (2001: 5), “determinadas características peculiares y diversos fallos de mercado justifican una regulación pública de este sector que altera los resultados que se alcanzarían en un equilibrio de libre mercado. Esta intervención incluye generalmente el acceso al mercado, el establecimiento de niveles de servicios mínimos justificados por obligaciones de servicio público y el control de los precios".

Evidentemente, si admitimos que el servicio y las rutas vienen impuestas, la entrada al mercado restringida, los precios controlados, la existencia de trayectos no rentables que se deben mantener por cuestiones de beneficio social, etc., podemos convenir que estamos ante un sector fuertemente regulado, a nivel local, regional, nacional e incluso comunitario.

No obstante, a pesar de esta regulación, desde mediados del pasado siglo se quería fomentar por parte de las autoridades españolas el acceso al sector del transporte, normalmente deficitario. A título indicativo, en la extinta Ley sobre ordenación de los transportes mecánicos por carretera de 27 de diciembre de 1947 se decía textualmente que se pretendía "abrir más ancho cauce a la iniciativa privada".

En el último cuarto del siglo XX, cuando se aprobó el Reglamento Nacional de los servicios urbanos e interurbanos de transportes en automóviles ligeros, se estableció que los municipios con área de influencia recíproca y consecuentemente, interacción de tráfico, deberán coordinarse en fórmula jurídica adecuada para la prestación de los servicios, siempre bajo la atenta mirada de la Administración Pública.

En el ámbito de las Corporaciones Locales, la Ley 7/1985 de 2 de abril, reguladora de las bases del régimen local, señala en su artículo 25.2 que el municipio ejercerá competencias en diferentes materias entre las cuales se incluye el transporte público de viajeros.

La gestión de estos servicios puede hacerse de forma directa o indirecta y en la citada ley se indican como posibles, las siguientes:

- La gestión directa adoptará alguna de las siguientes formas:

- Gestión por la propia entidad local

- Organismo autónomo local

- Sociedad mercantil, cuyo capital social pertenezca íntegramente a la entidad local

- La gestión indirecta, por su parte, adoptará alguna de las siguientes formas:

- Concesión

- Gestión interesada

- Concierto 


\section{- Arrendamiento}

- Sociedad mercantil y cooperativas legalmente constituidas cuyo capital pertenezca sólo parcialmente a la entidad local.

La gestión directa es una de las figuras más utilizadas por las corporaciones locales, especialmente en lo que se refiere a la prestación del servicio de transporte urbano de viajeros. Tal es el caso de ciudades como Madrid, Barcelona, Málaga, Sevilla, Las Palmas de Gran Canaria, etc.

No obstante, la gestión indirecta adquiere cada vez mayor relevancia, ocupando la concesión administrativa un lugar preeminente. Este es el caso de ciudades como Zaragoza, A Coruña, Girona, Granada, Bilbao, etc.

En cualquier caso, serán las corporaciones locales las que autorizarán la creación, implantación y prolongación de servicios urbanos de transporte de viajeros, siendo necesario un informe de la administración pertinente en caso de coincidencia con el transporte interurbano de forma que se establezca la adecuada coordinación.

En otro orden de cosas, si partimos de tres ejes esenciales a tener en cuenta en el desarrollo de las ciudades -ciudadanos, movilidad y urbanismo- podemos advertir los problemas principales a los que actualmente se enfrenta el sector del transporte urbano, objeto de debate tanto entre los gestores de las empresas que prestan el servicio como entre las administraciones públicas responsables de la prestación del mismo.

Entre las cuestiones más suscitadas cabe destacar aquellas que giran en torno a la inversión en infraestructura y material móvil o la financiación de las empresas del sector, en la que los gestores llevan realizando especial hincapié en los últimos años.

No cabe duda que la crisis ha puesto en tela de juicio los tradicionales sistemas y formas de gestión de recursos, así como las opciones de financiación del sector público y privado español, viéndose afectado también, de forma directa e indirecta, el sector que nos ocupa. Las continuas políticas de recortes para reducir el gasto público, unidas a cambios en la forma de movilidad en las ciudades, afectan a los ingresos de las operadoras del sector, siendo imprescindible realizar ajustes de gastos y costes, sin que ello repercuta negativamente en la calidad del servicio. En suma, se requiere una gestión eficaz y eficiente con un férreo control de costes.

En este contexto, el referido servicio de transporte regular urbano atraviesa por continuas necesidades financieras con una escasa rentabilidad económica, como así se evidencia en el Informe del Observatorio del Transporte Urbano Colectivo' (2013), al llegar a conclusiones como las siguientes:

- Existen empresas que parecen reflejar una imagen de inestabilidad financiera, derivada de un abuso de fuentes de financiación a corto plazo o un exceso de activos inmovilizados.

- El indicador del endeudamiento del colectivo refleja un valor medio en torno al $60 \%$, encontrando empresas con ratio de endeudamiento superior al $90 \%$ de su estructura financiera.

- Hay empresas cuya cifra de negocios no permite cubrir ni tan siquiera los gastos económicos derivados del desarrollo de la actividad.

- Por término medio, el colectivo arroja pérdidas en el resultado financiero.

- La mayor parte del colectivo de empresas arroja rentabilidades positivas a la hora de gestionar sus recursos económicos, con un ligero descenso en los valores indicadores, alcanzando en 2011 una rentabilidad económica del 3,03\%.

En muchas ocasiones, tal situación económico-financiera se atribuye al carácter público que ostenta este servicio, principalmente en municipios de más de 50.000 habitantes. A tenor del artículo 26 de la mencionada Ley 7/1985 de 2 de abril, reguladora de las bases de régimen local, tales municipios por sí mismos o asociados deberán prestar, en todo caso, el servicio del transporte colectivo urbano de viajeros.

Como se señala en el mencionado Informe del Observatorio (2013), dado el techo del gasto de las Administraciones Públicas, "se tendrán que buscar fuentes de financiación alternativas que paulatinamente sustituyan las aportaciones de las Administraciones Públicas, debido a las restricciones en el gasto que limitan las posibilidades por parte de las empresas operadoras de obtener subvenciones para el servicio, al considerar como prioridad absoluta el pago de intereses y capital de la deuda pública."

$1 \quad$ El objetivo fundamental de este Observatorio es el estudio de las condiciones de explotación de las empresas de transporte urbano colectivo en superficie, realizando publicaciones bienales sobre su situación económico-financiera, estructura de costes y financiación. 
Conseguir un sistema de financiación para el sector, eficiente y efectivo, es sin duda fundamental, pero tanto como la continua mejora de la propia gestión interna del servicio. A este último orden se adhiere nuestro trabajo, en el que pretendemos establecer el porcentaje de empresas eficientes de las operadoras en España del servicio de transporte urbano colectivo en superficie durante el período 2008-2011, tanto a nivel global del sector como por segmentos relacionados con el tamaño de la organización.

Cabe señalar la existencia de otros aspectos también polémicos y de interés en este ámbito, relacionados con la propia actividad del transporte, entre ellos la congestión de las ciudades, derivada del reparto del uso viario, el consumo de energía, la emisión de gases de efecto invernadero o la emisión de otros contaminantes, como el plomo, monóxido de carbono, óxido de azufre, nitrógeno, etc.

La solución a estas cuestiones pasa obviamente por una combinación de medidas que combine la racionalización de los precios del transporte, la adquisición de material móvil más amable con el medio ambiente, menos contaminante, y por una ley nacional de financiación del transporte público, ya que sólo unas pocas comunidades autónomas disponen de regulación en este aspecto.

A nuestro juicio, queda justificada la curiosidad de realizar estudios sobre el sector del transporte urbano de pasajeros², los cuales deben permitir, entre otras cuestiones, ahondar en la mejora de su situación económicofinanciera, sin dejar de prestar un servicio a unas tarifas sostenibles, desde una perspectiva económica y social. El presente trabajo pretende facilitar la reflexión a gestores, responsables políticos, investigadores y ciudadanos en general a través del análisis de la eficiencia de las empresas de transporte urbano colectivo en España.

En términos generales, la eficiencia de una organización alude a la capacidad de articular convenientemente los inputs utilizados con los outputs obtenidos. En otras palabras, obtener el máximo volumen de producción, en su sentido más amplio, al mínimo coste posible, resultando consiguientemente el mejor rendimiento, técnico y/o económico, de sus factores productivos y centros de actividad.

Es indudable el interés que suscita evaluar la eficiencia de una unidad productiva, más aún cuando para el desarrollo de su actividad se requiera la utilización de recursos de índole pública. Interés que se convierte en prácticamente una obligación cuando nos encontramos en un entorno de crisis económica y financiera como la que se vive en España desde 2007. En suma, el presente trabajo puede resultar de interés para contribuir a una gestión más eficiente de la explotación del servicio público de transporte.

\section{EVALUACIÓN DE LA EFICIENCIA}

De entre las primeras medidas cuantitativas del concepto de eficiencia se encuentra la de Farrell (1957: 259), que distingue la eficiencia técnica y la asignativa (precios). Una empresa será eficiente técnicamente si no puede producir o prestar más servicio (generar más output) dado un conjunto de factores productivos (inputs), haciendo referencia la eficiencia en precios a elegir la combinación óptima de factores (fabricar el output o prestar el servicio al coste menor).

Sea la eficiencia global, técnica o económica, su medida implica tomar de referencia un standard respecto al cual cuantificarla. De una parte, se puede obtener una medida comparando el quehacer de la empresa con respecto a lo que en ella misma se hubiere fijado en la cuantificación previa de los objetivos de la organización, bajo el paraguas del control presupuestario, obteniendo indicadores ya sea a nivel de los factores productivos consumidos, o bien de los centros y/o actividades en los que haya sido convenientemente estructurada. Otra forma de cuantificar la eficiencia es a través de la utilización de modelos frontera, considerando la realidad de la empresa con la del conjunto de su sector.

En este último contexto se ha venido utilizando en la literatura modelos con distintas perspectivas que, tal como se recoge en la Tabla 1, pudieran clasificarse atendiendo, entre otros, a dos criterios: contemplar o no una forma específica de la función frontera y el ruido estadístico.

2 Si bien existe una amplia gama de estudios sobre la evaluación de la eficiencia en distintos sectores empresariales, inclusive en el sector del transporte, la literatura específica en el ámbito del transporte urbano de pasajeros por carretera en España es mucho más limitada. 
TABLA 1

TIPOLOGÍA DE MODELOS PARA LA EVALUACIÓN DE LA EFICIENCIA

\begin{tabular}{|c|c|c|}
\hline CRITERIO & MODELO & CARACTERÍSTICA \\
\hline \multirow{2}{*}{ FUNCIÓN FRONTERA } & PARAMÉTRICO & Se asume una forma funcional \\
\cline { 2 - 3 } & NO PARAMÉTRICO & No se asume una forma funcional específica \\
\hline \multirow{2}{*}{$\begin{array}{c}\text { RUIDO } \\
\text { ESTADÍSTICO }\end{array}$} & DETERMINISTA & No contempla existencia ruido estadístico \\
\cline { 2 - 3 } & ESTOCÁSTICO & Contempla existencia ruido estadístico \\
\hline
\end{tabular}

Fuente: Elaboración propia.

A nuestro juicio, ninguna de las alternativas planteadas puede considerarse excluyente en el estudio de la eficiencia de una unidad productiva, porque aunque si bien es verdad que el análisis no frontera posee quizás menor rigor econométrico o matemático, enriquece muchísimo la gestión práctica de una organización, a través del control de su propia gestión. En este trabajo, nos centraremos en la evaluación del grado de eficiencia de las empresas de transporte urbano de pasajeros a través de los modelos que construyen una frontera, sin asumir una función específica, respecto a la cual medir la eficiencia, utilizando un enfoque no paramétrico determinista, como es el Análisis Envolvente de Datos (DEA).

Utilizando los modelos fronteras podemos encontrar distintos trabajos sobre el sector del transporte en España, como son los de Matas y Raymond (1998), referido a 9 compañías en el período 1983-1995, Pina y Torres (2000), que se circunscribe al transporte urbano de una región española, o Castelló y Talliani (2008), referido al transporte de mercancías, entre otros.

Los modelos DEA parten de establecer un conjunto de variables que son su fuente de alimentación (inputs y outputs) para un conjunto de DMU (Decision Making Units), determinando la mejor práctica de la muestra representativa del universo poblacional, y, a partir de ahí, el grado de eficiencia versus ineficiencia de cada DMU. En nuestro caso tomaremos como Unidad de toma de decisión cada empresa operadora del servicio en España. Por consiguiente, para cada una de ellas se obtendrá su medida del grado de eficiencia, que nos permitirá determinar el porcentaje de empresas más o menos eficientes respecto a la frontera de la muestra analizada, así como los correspondientes potenciales de mejora.

Es factible cuantificar la eficiencia a través de la medición del gap existente entre los outputs de una unidad productiva y los de la "mejor" empresa de su sector, para unos inputs dados (modelo orientado a outputs), o bien la que se deriva de comparar los inputs utilizados respecto al nivel de outputs dados (modelo orientado a inputs). De ello se deduce que con el análisis de eficiencia a través de los modelos DEA no se pretende fijar el estándar ideal, máximo o teórico para operar en el sector sino que este se sustituye por el que ostentan las mejores empresas consideradas en el estudio.

De otra parte, la forma de la frontera de producción guarda relación con los rendimientos a escala. A este respecto, encontramos en la literatura el DEA desarrollado con rendimientos a escala constantes (CRS) que posteriormente se extiende hacia situaciones de rendimientos variables (VRS), permitiendo diferenciar en la eficiencia técnica global la puramente técnica y la eficiencia a escala, métodos que son descritos en Fare, Grosskopf y Lovell $(1994)^{3}$.

En este trabajo se ha optado por utilizar un modelo DEA con rendimientos variables a escala, reduciéndose de este modo el efecto de la escala en la selección de las empresas que determinan la frontera de eficiencia. Además, de las dos opciones que permite este análisis, se ha elegido la opción de minimización de los inputs con el output existente (Modelo orientado a inputs).

Como resultado de aplicar el modelo DEA comentado sobre las empresas de transporte urbano de la muestra, se obtiene la denominada clasificación de eficiencia. Dicha clasificación presenta, por un lado, un listado de las empresas analizadas con sus correspondientes niveles de eficiencia que nos conduce a una categorización de los resultados obtenidos.

\footnotetext{
3 Citado en Coelli (2008).
} 
El análisis DEA permite además determinar las mejores prácticas dentro de la muestra analizada, esto es, aquellas empresas que se pueden tomar como referencia externa. El resto de las mismas para acceder a la frontera de eficiencia tendrían que acometer algunas mejoras. Del programa de análisis DEA utilizado para el estudio se puede obtener un resumen de los potenciales de mejora que, con relación a los inputs y outputs seleccionados, presentan las empresas que no han alcanzado un nivel de eficiencia del 100\%. Dichos porcentajes se obtienen para el total de la muestra como suma de las mejoras potenciales parciales que se podrían llevar a cabo, no siendo más que la expresión cuantitativa de la disminución de inputs o aumento factible de outputs.

\section{SELECCIÓN DE LAS DMU}

La muestra representativa del sector objeto de análisis está configurada por 42 DMU (empresas prestatarias del servicio de transporte urbano de viajeros en diferentes provincias del territorio español). Todas ellas han presentado los estados contables de los ejercicios 2008 a 2011 en los Registros Mercantiles correspondientes, y han superado una serie de filtros y controles que fueron establecidos, principalmente con el propósito de su homogeneización en cuanto a que su actividad esencial fuera el transporte urbano de viajeros así como que en el municipio en que opere tenga más de 50.000 habitantes $^{4}$.

Definitivamente, tras la aplicación de las correspondientes restricciones, para el período objeto de estudio (2008-2011), la muestra queda repartida por Comunidades Autónomas de la forma que se muestra en la Tabla 2, quedando representadas concesionarias de todos los tamaños y de las distintas formas de titularidad jurídica existentes. Se ofrece un panorama representativo de las empresas del sector, toda vez que el conjunto de entidades presta servicio a cerca del $60 \%$ de la población total que vive en ciudades de más de 50.000 habitantes.

TABLA 2

DISTRIBUCIÓN DE LA MUESTRA POR COMUNIDADES AUTÓNOMAS

\begin{tabular}{|c|c|c|c|}
\hline CC.AA/ Ciudad Autónoma & $\mathrm{N}^{\circ}$ Empresas & CC.AA/Ciudad Autónoma & $\mathrm{N}^{\circ}$ Empresas \\
\hline Andalucía & 9 & Aragón & 1 \\
\hline 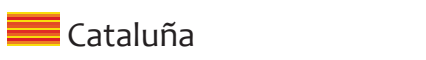 & 5 & Extremadura & 1 \\
\hline$\ldots$ Com. de Madrid & 2 & Islas Baleares & 1 \\
\hline$\equiv$ Com. Valenciana & 6 & + Princip.de Asturias & 3 \\
\hline Galicia & 3 & Navarra & 0 \\
\hline 鱼总 Castilla y León & 4 & Cantabria & 0 \\
\hline Euskadi & 2 & La Rioja & 1 \\
\hline Canarias & 2 & $\mathbf{1}$ Ceuta & 0 \\
\hline 밈 Castilla-La Mancha & 1 & Melilla & 0 \\
\hline Región de Murcia & 1 & TOTAL & 42 \\
\hline
\end{tabular}

Fuente: Observatorio TUC (2013).

Como puede observarse en la Tabla 3, y se recoge en el citado Informe del Observatorio del Transporte Urbano Colectivo, se trata de empresas que podríamos caracterizar en la siguiente forma:

- Constituidas en su mayor parte hace más de 10 años. "Probablemente, en ello ha tenido una incidencia significativa el hecho de estar ante un sector excesivamente regulado; se legislan aspectos tales como la fijación de tarifas, el acceso al mercado, las fórmulas de gestión, la integración de redes de transporte, los itinerarios, las paradas, la adquisición y el uso de infraestructuras, etc. A todo esto se añade la excesiva dependencia de subvenciones públicas al verse obligadas, en la inmensa mayoría de las ocasiones, a la prestación de servicios no rentables, motivado por cuestiones eminentemente sociales. Es cierto que si bien la obtención de subvenciones de la Administración Pública supone unos ingresos garantizados, la

4 Se ha utilizado la misma muestra de empresas configurada para los informes del ya citado Observatorio, con sus mismos filtros y controles, relacionados con la disponibilidad de las cuentas, número mínimo de empleados, estado, habitantes del municipio y su actividad principal. A este respecto puede verse el segundo informe de dicho Observatorio (2013:45). 
demora que caracteriza a la Administración en el pago de las mismas desmotiva a los capitales privados a competir en y por el sector". (OTUC, 2013: 47).

- En cuanto a la forma jurídica, la mayor parte $(76,19 \%)$ “está conformada por sociedades anónimas, consecuencia de la estructura empresarial del sector, donde es muy habitual la existencia de empresas municipales de transporte. Son básicamente sociedades anónimas municipales las utilizadas por los ayuntamientos para prestar el servicio en los municipios mayores, copando éstas la mayor parte del negocio" (OTUC, 2013: 48).

- Respecto a la titularidad jurídica, debemos precisar en primer lugar que, tal como se ha comentado con anterioridad, en España la gestión del servicio que nos ocupa puede hacerse de forma directa por la Corporación Local o gestionarse de forma indirecta.

De las DMU (Decision Making Units) que configuran la muestra, la titularidad jurídica presenta un comportamiento más dispar. El 38,09\% de las empresas son públicas y el 57,14\% privadas, siendo la dimensión promedio mayor en el caso de las públicas que en el de las privadas que, generalmente, se responsabilizan del servicio en los municipios más pequeños.

- Con relación al número de trabajadores, más del 75\% de las empresas tienen menos de 500 trabajadores.

TABLA 3

CARACTERÍSTICAS DE LA MUESTRA.

\begin{tabular}{|c|c|c|c|c|c|}
\hline Criteri & os de clasificación & Número de em- & Porcentaje & $\begin{array}{l}\text { Cifra de nego- } \\
\text { cios media }\end{array}$ & $\begin{array}{c}\text { Fondos propios } \\
\text { medios }\end{array}$ \\
\hline & Más de 10 años & 38 & $90,48 \%$ & $30.467,94$ & $13 \cdot 343,20$ \\
\hline Antigüedad & Entre 5 y 10 años & 2 & $4,76 \%$ & $1.955,50$ & $1.277,25$ \\
\hline & Entre 1 y 5 años & 2 & $4,76 \%$ & $6.504,51$ & $-1.039,62$ \\
\hline & Total & 42 & $100,00 \%$ & $27.255,48$ & $11.622,13$ \\
\hline & Sociedades Anónimas & 32 & $76,19 \%$ & $33.663,96$ & $13.103,67$ \\
\hline Personalidad & Sociedades Limitadas & 10 & $23,81 \%$ & $6.748,37$ & $7.029,36$ \\
\hline Jurídica & Otras figuras & - & - & - & - \\
\hline & Total & 42 & $100,00 \%$ & $27.255,48$ & $11.622,13$ \\
\hline & Pública & 16 & $38,09 \%$ & $51.946,85$ & $19.133,45$ \\
\hline Titularidad & Privada & 24 & $57,14 \%$ & $12.809,70$ & $7.842,98$ \\
\hline & Mixta & 2 & $4,77 \%$ & $3.073,97$ & 637,07 \\
\hline & Total & 42 & $100,00 \%$ & $27.255,48$ & $11.622,13$ \\
\hline & Inferior a 250 & 25 & $59,52 \%$ & $5.804, .90$ & $2.682,49$ \\
\hline & Entre 250 y 500 & 7 & $16,67 \%$ & $17.649,52$ & 14.772 .33 \\
\hline $\begin{array}{l}\text { Número de tra- } \\
\text { bajadores }\end{array}$ & Entre 501 y 1.000 & 5 & $11,90 \%$ & $20.828,18-$ & 669,87 \\
\hline & Superior a 1.000 & 5 & $11,90 \%$ & $154 \cdot 384,04$ & $63.492,34$ \\
\hline & Total & 42 & $100,00 \%$ & $27.255,48$ & $11.622,13$ \\
\hline
\end{tabular}

Fuente: Adaptada de II Informe Observatorio TUC (2013).

\section{SELECCIÓN DE VARIABLES}

El primer paso en el Análisis Envolvente de Datos lo constituye la selección de las variables inputs y outputs que se van a contemplar en el análisis multidimensional de la eficiencia de la muestra. Es la elección de las variables 
un tema fundamental para la robustez y validez de las conclusiones a obtener. Ahora bien, concretar los inputs y outputs, las fuentes de las que se alimenta el Modelo, no resulta fácil en un sector en el que se carece de información pública de nociones claves como son los kilómetros recorridos, viajeros transportados u otras variables de índole técnica.

Por ello, de una parte, definimos como recursos o indicadores de entrada a las unidades de medida que representan los principales factores productivos que se utilizan para llevar a cabo la prestación de servicios. Ellos son los criterios utilizados para evaluar la economía y la eficiencia de los programas y servicios. Dado un cierto nivel de actividad, la mejora de la eficiencia requiere una disminución del consumo de estos elementos, cuales son de índole material, técnico o humano. Así pues, la selección de los inputs o factores que influyen sobre los outputs fue la de aquellos gastos de explotación que determinan, tanto la actividad desarrollada por la empresa como la dimensión de su activo productivo, optándose por los siguientes: "Aprovisionamientos", "Gastos de personal”, “Otros gastos de explotación” y “Amortización del inmovilizado".

Por su parte, los indicadores de producto miden el rendimiento o el nivel de actividad de los programas y servicios. Podrían establecerse diferentes clasificaciones, centrándose en los objetivos de la organización, que proporciona una medida de eficacia, o, podrían centrarse en la actividad llevada a cabo por la organización, tales como la productividad y el nivel de servicio. Dado que el objetivo fundamental planteado para este estudio es la eficiencia de las operaciones de explotación, se selecciona, como output representativo de la eficiencia operativa a analizar los ingresos de explotación, siendo la variable que los va a definir en el modelo el "importe neto de la cifra de negocios".

No obstante lo anterior, cabe advertir que en España la cifra de negocios de las operadoras del servicio está afectada por las condiciones de financiación del mismo. Así, como señala Suárez (2004: 101), "si la Administración fija, por motivos económicos o sociales, tarifas oficiales que no permitan lograr un equilibrio económico a las em-

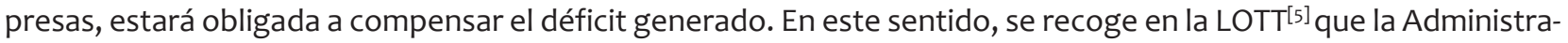
ción no debe cubrir los déficits generados por una gestión inadecuada, pero como en la mayor parte de las ocasiones es difícil determinar qué parte se debe a esa deficiente gestión, es habitual financiar la totalidad del déficit".

El problema que se plantea es que las aportaciones públicas vinculadas con la prestación del servicio no se ofrecen bajo un mismo concepto ni tienen igual tratamiento contable, toda vez que, como se sintetiza en el Informe del Observatorio del Transporte Urbano Colectivo (2013: 141), aglutinan:

1. "Subvenciones en tarifas, que las empresas suelen registrar junto a la recaudación directa, como cifra de negocios de su actividad.

2. Subvenciones a la explotación vía contratos programas, que determinadas empresas reciben, en función de una serie de parámetros determinados, para sufragar los principales gastos de explotación del servicio, que son: déficit tarifario y gastos por amortizaciones de inversiones.

3. Otras subvenciones por compensación de déficit, subvenciones generales de explotación que las empresas reciben para compensar las pérdidas de explotación por la falta de cobertura de sus gastos de explotación a través de su recaudación. En estos casos, no se hace una distinción entre la compensación de déficit en tarifas y la compensación de gastos generales.

4. Aportación de socios para compensación de déficit, cuando directamente se recibe una aportación económica de los socios de la empresa que se computa directamente en el patrimonio, aunque su finalidad es, como en los casos anteriores, la cobertura de los resultados negativos obtenidos."

Por tanto, en los niveles de eficiencia o ineficiencia de la empresa resultantes del análisis envolvente de datos inciden en mayor o menor medida la forma de obtención de la subvención para la cobertura del déficit, al poder estar integrada en la cifra de negocios de la empresa. Pero esto no deja de ser más que un reflejo de la propia realidad del sector español con las consiguientes consecuencias económicas en los resultados de la gestión de las empresas. Teniendo en cuenta tal característica, adaptamos en nuestro trabajo la discriminación adoptada por Castelló y Giralt (2008), distinguiendo los siguientes niveles:

- Empresas en la frontera de eficiencia

- Empresas marginalmente eficientes. Puntuación de eficiencia comprendida entre el 90\% y el 99,99\%.

$5 \quad$ Ley de Ordenación de los Transportes Terrestres (1987). 
- Empresas con niveles de eficiencia comprendidos entre el $80 \%$ y el $89,99 \%$

- Empresas con nivel de eficiencia menor al 80\%.

\section{ANÁLISIS DE LOS RESULTADOS A NIVEL GLOBAL}

Construido el modelo se aplica el DEA utilizando el programa "Data Envelopment Analisys (Computer) Program $^{6}$ " y se procede a la interpretación de los resultados de la clasificación de eficiencia, determinación de la mejor práctica de la muestra y al análisis de los potenciales de mejora.

Una de las salidas de información del Modelo DEA presenta el valor obtenido para cada firma, así como la media resultante, de la Eficiencia en el modelo CRS, en el modelo VRS, y la eficiencia de escala. En la Tabla 4 se recogen los resultados medios obtenidos a nivel global, apreciándose cierta estabilidad durante el período de estudio de los niveles de eficiencia. Así, la eficiencia global conjunta (CRS) es del 69\% en 2008, descendiendo hasta el 67,9\% en 2011. Tales cifras evidencian una posible reducción de los inputs considerados en aproximadamente un 31\% ó 32\%, sin cambiar el nivel de outputs. En otras palabras, técnicamente podría generarse la cifra de outputs con un $69 \%$ ó $67,9 \%$, según el año, de los inputs considerados. Todo ello con un ligero aumento de las empresas que se sitúan en la frontera de eficiencia CRS, que pasan de ser 11 en 2008 a 13 en 2011.

Si observamos la eficiencia técnica, acuñada en la literatura como eficiencia técnica "pura” (VRS), se aprecia que arroja un dato medio mayor que en la conjunta (CRS), con un resultado del 9\% ó 7\% de ineficiencia motivada por no estar en la escala considerada óptima. Las referidas ineficiencias de escala se originan porque en el sector operan empresas con rendimientos decrecientes y/o crecientes a escala, mostrando cómo un $42,85 \%$ del colectivo presenta rendimientos crecientes en 2008 frente al 45,23\% en 2011. Por su parte, son 12 empresas (28,57\% de la muestra) en 2008 y sólo 9 (21,43\%) en 2011 las que presentan rendimientos decrecientes.

TABLA 4

PROMEDIOS DE EFICIENCIA

\begin{tabular}{|l|r|r|r|r|}
\hline & \multicolumn{1}{|c|}{2008} & 2009 & 2010 & 2011 \\
\hline Eficiencia CRS & 0,690 & 0,680 & 0,683 & 0,679 \\
\hline Eficiencia VRS & 0,759 & 0,754 & 0,750 & 0,732 \\
\hline Eficiencia escala & 0,910 & 0,910 & 0,912 & 0,929 \\
\hline Número de empresas eficientes CRS & 11 & 11 & 12 & 13 \\
\hline Número de empresas eficientes VRS & 14 & 14 & 16 & 17 \\
\hline Número de empresas con drs & 12 & 7 & 12 & 9 \\
\hline Número de empresas con irs & & 24 & 18 & 19 \\
\hline
\end{tabular}

Fuente: Elaboración propia

En una primera aproximación, escogiendo las cifras que arroja la eficiencia VRS, por ser menos restrictiva que la CRS, y como puede observarse en la Tabla 5, en 2008 el 33,33\% de las empresas del colectivo se sitúan en la frontera de eficiencia, presentando casi el 55\% niveles inferiores al 80\% de eficiencia. Es decir, nos encontramos que poco más de la mitad del colectivo podría reducir sus inputs en más de un $20 \%$ para sus niveles de outputs. La situación en 2011 nos lleva a un mayor número de empresas que se sitúan en la frontera de eficiencia.

6 Una guía del mismo puede verse en Coelli (2008). 
TABLA 5

CLASIFICACIÓN GENÉRICA DEA DE EFICIENCIA DEL CONJUNTO DE LA MUESTRA

\begin{tabular}{|c|c|c|c|c|c|c|c|c|}
\hline & \multicolumn{2}{|c|}{2008} & \multicolumn{2}{|c|}{2009} & \multicolumn{2}{|c|}{2010} & \multicolumn{2}{|c|}{2011} \\
\hline & $\mathrm{N}^{\circ}$ & $\%$ & $\mathrm{~N}^{\circ}$ & $\%$ & $\mathrm{~N}^{0}$ & $\%$ & $\mathrm{~N}^{\circ}$ & $\%$ \\
\hline $\begin{array}{l}\text { Empresas eficientes. Puntuación de } \\
\text { eficiencia del } 100 \%\end{array}$ & 14 & $33,33 \%$ & 14 & $33,33 \%$ & 16 & $38,10 \%$ & 17 & $40,48 \%$ \\
\hline $\begin{array}{l}\text { Empresas marginalmente eficientes. } \\
\text { Puntuación de eficiencia comprendida } \\
\text { entre el } 90 \% \text { y el } 99,99 \%\end{array}$ & 3 & $7,14 \%$ & 5 & $11,90 \%$ & 3 & $7,14 \%$ & 1 & $2,38 \%$ \\
\hline $\begin{array}{l}\text { Puntuación de eficiencia comprendida } \\
\text { entre el } 80 \% \text { y el } 89,99 \%\end{array}$ & 2 & $4,76 \%$ & 2 & $4,76 \%$ & 0 & $0,00 \%$ & 2 & $4,76 \%$ \\
\hline Puntuación de eficiencia inferior al 80\% & 23 & $54,76 \%$ & 21 & $50,00 \%$ & 23 & $54,76 \%$ & 22 & $52,38 \%$ \\
\hline
\end{tabular}

Fuente: Elaboración propia

Con relación a los potenciales de mejora u holguras, como se aprecia en la Tabla 6, cabe señalar como más representativos en el año 2008 los "gastos de personal" y "otros gastos de explotación". En 2009, resulta significativo el aumento experimentado por el input "aprovisionamientos", de 3 a 8 empresas, así como la reducción de empresas que presenta holguras en la partida "otros gastos de explotación". Señalar que el input "gastos de personal" evidencia una tendencia decreciente a lo largo de todo el período de estudio respecto al número de empresas que arroja potenciales de mejora en este input. No obstante, aunque disminuye el porcentaje de número de holguras en "gastos de personal" sigue siendo el más significativo en 2011. Por su parte, la "amortización del inmovilizado" presenta un mayor número de holguras en 2011 respecto al primer año. Aunque pocas, existen empresas con potenciales de mejora en su cifra de negocios.

TABLA 6

POTENCIALES DE MEJORA EN EL CONJUNTO DE LA MUESTRA

\begin{tabular}{|c|c|c|c|c|c|c|c|c|}
\hline & \multicolumn{2}{|c|}{2008} & \multicolumn{2}{|c|}{2009} & \multicolumn{2}{|c|}{2010} & \multicolumn{2}{|c|}{2011} \\
\hline & $\mathrm{N}^{\circ}$ & $\%$ & $\mathrm{~N}^{\circ}$ & $\%$ & $\mathrm{~N}^{\circ}$ & $\%$ & $\mathrm{~N}^{\circ}$ & $\%$ \\
\hline Aprovisionamientos & 3 & $8,57 \%$ & 8 & $24,24 \%$ & 5 & $12,82 \%$ & 8 & $25,00 \%$ \\
\hline Gastos de personal & 16 & $45,71 \%$ & 14 & $42,42 \%$ & 14 & $35,90 \%$ & 13 & $40,63 \%$ \\
\hline Otros gastos de explotación & 11 & $31,43 \%$ & 4 & $12,12 \%$ & 11 & $28,21 \%$ & 3 & $9,38 \%$ \\
\hline Amortización del inmovilizado & 3 & $8,57 \%$ & 4 & $12,12 \%$ & 7 & $17,95 \%$ & 6 & $18,75 \%$ \\
\hline $\begin{array}{l}\text { Importe neto de la cifra de } \\
\text { negocios }\end{array}$ & 2 & $5,71 \%$ & 3 & $9,09 \%$ & 2 & $5,13 \%$ & 2 & $6,25 \%$ \\
\hline
\end{tabular}

Fuente: Elaboración propia

\section{ANÁLISIS DE LOS RESULTADOS SEGMENTADOS POR TAMAÑO}

Al objeto de profundizar en el análisis de la eficiencia técnica y aportar información añadida realizamos una segmentación de las empresas atendiendo a su tamaño, tomando como indicador del mismo la magnitud número de empleados, al ser la única variable técnica cuya información está disponible para la totalidad de la muestra. El situar las mismas empresas en cada uno de los años en tramos diferenciados idénticos debe permitirnos realizar un análisis dinámico. Se ha segmentado la muestra en cuatro grupos, siendo: 
Grupo 1: Empresas que disponen de menos de 250 trabajadores, a las que podríamos calificar como "pequeñas" (59,52\% de la muestra).

Grupo 2: Empresas con una plantilla comprendida entre 250 y 500 trabajadores, a las que podríamos calificar como "medianas pequeñas" (16,67\% de la muestra).

Grupo 3: Empresas con un número de trabajadores comprendido entre 501 y 1000 , a las que podríamos calificar como "medianas grandes" (11,90\% de la muestra).

Grupo 4: Empresas que disponen de más de 1000 trabajadores, a las que podríamos calificar como "grandes" (11,90\% de la muestra).

\section{Grupo 1. "Pequeñas".}

Como se observa en la Tabla 7, en el año 2008 sólo el 52\% de las empresas se encuentra en un nivel de eficiencia superior al $80 \%$, pasando a ser un $56 \%$ en 2011. El número de empresas que se sitúan en la frontera de eficiencia presenta una evolución positiva, a pesar del descenso en 2009. Por otra parte, cabe señalar que de las empresas de este grupo la horquilla del porcentaje de empresas que no presentan ineficiencias de escala se sitúa entre el $28 \%$ y el 40\%, delimitada por los valores de 2009 y 2011, respectivamente.

TABLA 7

CLASIFICACIÓN GENÉRICA DEA DE EFICIENCIA EMPRESAS GRUPO 1

\begin{tabular}{|l|r|r|r|r|}
\hline & 2008 & 2009 & 2010 & 2011 \\
\cline { 2 - 5 } & $\%$ & $\%$ & $\%$ & $\%$ \\
\hline $\begin{array}{l}\text { Empresas eficientes. Puntuación de eficiencia } \\
\text { del 100\% }\end{array}$ & $36,00 \%$ & $32,00 \%$ & $44,00 \%$ & $48,00 \%$ \\
\hline $\begin{array}{l}\text { Empresas marginalmente eficientes. Puntua- } \\
\text { ción de eficiencia comprendida entre el 90\% y } \\
\text { el 99,99\% }\end{array}$ & $12,00 \%$ & $20,00 \%$ & $12,00 \%$ & - \\
\hline $\begin{array}{l}\text { Puntuación de eficiencia comprendida entre el } \\
80 \% \text { y el 89,99\% }\end{array}$ & $4,00 \%$ & $8,00 \%$ & - & $8,00 \%$ \\
\hline Puntuación de eficiencia inferior al 80\% & $48,00 \%$ & $40,00 \%$ & $44,00 \%$ & $44,00 \%$ \\
\hline
\end{tabular}

Fuente: Elaboración propia

De otra parte, como se deduce de la Tabla 8, si bien en 2008 resalta como variable input con más número de holguras la de "gastos de personal”, seguida de los “otros gastos de explotación”, en el año siguiente es el factor "aprovisionamientos" el más significativo a este mismo, suponiendo un 33,33\% de los potenciales de mejora de ese período. En 2010, se configuran las “amortizaciones" como input más relevante respecto al número de empresas que presentan potencial reducción del mismo, mientras que en 2011, vuelven a ser los "aprovisionamientos". Aparentemente en el período de estudio ha ido perdiendo representatividad la variable "gasto de personal" si nos ceñimos al número de holguras, toda vez que aunque continúa presentando potenciales de mejora, su peso ha 
TABLA 8

POTENCIALES DE MEJORA EMPRESAS GRUPO 1

\begin{tabular}{|l|r|r|r|r|}
\hline & 2008 & 2009 & 2010 & 2011 \\
\cline { 2 - 5 } & $\%$ & $\%$ & $\%$ \\
\hline Aprovisionamientos & $15,79 \%$ & $33,33 \%$ & $25,00 \%$ & $38,89 \%$ \\
\hline Gastos de personal & $36,84 \%$ & $26,67 \%$ & $25,00 \%$ & $22,22 \%$ \\
\hline Otros gastos de explotación & $21,05 \%$ & $13,33 \%$ & $15,00 \%$ & $11,11 \%$ \\
\hline $\begin{array}{l}\text { Amortización del inmoviliza- } \\
\text { do }\end{array}$ & $15,79 \%$ & $13,33 \%$ & $30,00 \%$ & $22,22 \%$ \\
\hline $\begin{array}{l}\text { Importe neto de la cifra de } \\
\text { negocios }\end{array}$ & $10,53 \%$ & $13,33 \%$ & $5,00 \%$ & $5,56 \%$ \\
\hline
\end{tabular}

Fuente: Elaboración propia

\section{Grupo 2. "Medianas pequeñas"}

Como se observa en la Tabla 9, un 42,86\% de las empresas analizadas encuadradas dentro del grupo "medianas pequeñas" se consideran con niveles de eficiencia superiores al $80 \%$ en 2008 , cayendo este porcentaje al $28,58 \%$ en 2011. Resulta, pues, destacable cómo alrededor del 71\% de las empresas a partir de 2009 presenta niveles de eficiencia inferiores al $80 \%$, lo que implica que podrían obtener sus niveles de output con una reducción de más del 30\% en los inputs. Si observamos el indicador de ineficiencias de escala se constata que si bien en 2008 el $42,86 \%$ de las empresas presenta valores del 100\% en el mismo, este porcentaje disminuye hasta el 14,29\% en 2011.

TABLA 9

CLASIFICACIÓN GENÉRICA DEA DE EFICIENCIA EMPRESAS GRUPO 2

\begin{tabular}{|c|c|c|c|c|}
\hline & 2008 & 2009 & 2010 & 2011 \\
\hline & $\%$ & $\%$ & $\%$ & $\%$ \\
\hline $\begin{array}{l}\text { Empresas eficientes. Puntuación de eficiencia } \\
\text { del } 100 \%\end{array}$ & $28,57 \%$ & $28,57 \%$ & $28,57 \%$ & $14,29 \%$ \\
\hline $\begin{array}{l}\text { Empresas marginalmente eficientes. Puntua- } \\
\text { ción de eficiencia comprendida entre el } 90 \% \text { y } \\
\text { el } 99,99 \%\end{array}$ & - & - & - & $14,29 \%$ \\
\hline $\begin{array}{l}\text { Puntuación de eficiencia comprendida entre el } \\
80 \% \text { y el } 89,99 \%\end{array}$ & $14,29 \%$ & - & - & - \\
\hline Puntuación de eficiencia inferior al 80\% & $57,14 \%$ & $71,43 \%$ & $71,43 \%$ & $71,43 \%$ \\
\hline
\end{tabular}

Fuente: Elaboración propia

Con relación a los potenciales de mejora (Tabla 10) señalar que los más significativos respecto al número de empresas que presentan holguras en los inputs utilizados son, fundamentalmente, los "gastos de personal" y los "otros gastos de explotación". No hay empresas que presenten potencial de mejora en "aprovisionamientos", en los "otros gastos de explotación" ni en la "amortización del inmovilizado" en dos de los períodos. Resulta significativo que en 2011 más del 70\% de potenciales de mejora corresponden con "gastos de personal”, conociendo que este factor toma un valor de la mediana superior al $83 \%$ de los gastos de explotación, respecto a la cifra de negocios en las empresas del sector. 
TABLA 10

POTENCIALES DE MEJORA EMPRESAS GRUPO 2

\begin{tabular}{|c|c|c|c|c|}
\hline & 2008 & 2009 & 2010 & 2011 \\
\hline & $\%$ & $\%$ & $\%$ & $\%$ \\
\hline Aprovisionamientos & - & $25,00 \%$ & - & $14,29 \%$ \\
\hline Gastos de personal & $83,33 \%$ & $62,50 \%$ & $71,43 \%$ & $71,43 \%$ \\
\hline $\begin{array}{l}\text { Otros gastos de explo- } \\
\text { tación }\end{array}$ & $16,67 \%$ & - & $28,57 \%$ & \\
\hline $\begin{array}{l}\text { Amortización del inmo- } \\
\text { vilizado }\end{array}$ & & $12,50 \%$ & & $14,29 \%$ \\
\hline $\begin{array}{l}\text { Importe neto de la cifra } \\
\text { de negocios }\end{array}$ & & & & \\
\hline
\end{tabular}

Fuente: Elaboración propia

\section{Grupo 3. "Medianas grandes"}

Como se aprecia en la Tabla 11, la totalidad de empresas que no se sitúan en la frontera de eficiencia obtienen unos niveles de eficiencia inferiores al $80 \%$ en todos los años analizados, con una total estabilidad en cuanto al número de empresas que se sitúa como referente en la frontera de este grupo. Destaca que en este grupo de empresas no hay ninguna que no presente algún valor de ineficiencias de escala. Por tanto, hay un porcentaje de empresas cuya eficiencia pura es del $100 \%$, pero no su eficiencia a nivel global.

TABLA 11

CLASIFICACIÓN GENÉRICA DEA DE EFICIENCIA EMPRESAS GRUPO 3

\begin{tabular}{|l|r|r|r|r|}
\hline & 2008 & 2009 & 2010 & 2011 \\
\cline { 2 - 5 } & $\%$ & $\%$ & $\%$ & $\%$ \\
\hline $\begin{array}{l}\text { Empresas eficientes. Puntuación de } \\
\text { eficiencia del 100\% }\end{array}$ & $20,00 \%$ & $20,00 \%$ & - & $20,00 \%$ \\
\hline $\begin{array}{l}\text { Empresas marginalmente eficientes. } \\
\text { Puntuación de eficiencia comprendida } \\
\text { entre el 90\% y el 99,99\% }\end{array}$ & & - & - & - \\
\hline $\begin{array}{l}\text { Puntuación de eficiencia comprendida } \\
\text { entre el 80\% y el 89,99\% }\end{array}$ & & & - & - \\
\hline Puntuación de eficiencia inferior al 80\% & $80,00 \%$ & $80,00 \%$ & & \\
\hline
\end{tabular}

Fuente: Elaboración propia

Para este grupo, los potenciales de mejora que se observan (Tabla 12) como más significativos son los "gastos de personal” y los “otros gastos de explotación”. En 2011, disminuye el número de holguras en este último input en favor de las de "gastos de personal". No hay empresas que presenten potencial de mejora en "aprovisionamientos", ni en el input de "amortización del inmovilizado". Tampoco ninguna empresa de este grupo presenta potenciales de mejora en la cifra de negocios. 
TABLA 12

POTENCIALES DE MEJORA EMPRESAS GRUPO 3

\begin{tabular}{|c|c|c|c|c|}
\hline & 2008 & 2009 & 2010 & 2011 \\
\hline & $\begin{array}{l}\mathrm{N}^{\circ} \\
\%\end{array}$ & $\begin{array}{l}\mathrm{N}^{\circ} \\
\%\end{array}$ & $\begin{array}{l}\mathrm{N}^{\circ} \\
\%\end{array}$ & $\begin{array}{l}\mathrm{N}^{\circ} \\
\%\end{array}$ \\
\hline Aprovisionamientos & - & - & - & - \\
\hline Gastos de personal & $50,00 \%$ & $80,00 \%$ & $50,00 \%$ & $75,00 \%$ \\
\hline $\begin{array}{l}\text { Otros gastos de explo- } \\
\text { tación }\end{array}$ & $50,00 \%$ & $20,00 \%$ & $50,00 \%$ & $25,00 \%$ \\
\hline $\begin{array}{l}\text { Amortización del inmo- } \\
\text { vilizado }\end{array}$ & - & - & - & - \\
\hline $\begin{array}{l}\text { Importe neto de la cifra } \\
\text { de negocios }\end{array}$ & - & - & - & - \\
\hline
\end{tabular}

Fuente: Elaboración propia

\section{Grupo 4. "Grandes".}

En todos los años analizados, el porcentaje de empresas que se sitúa en la frontera de eficiencia es del 40\%, frente al 60\% con niveles de eficiencia inferiores al 80\%, a excepción de en el año 2009, que la situación es justo la contraria, aumentan considerablemente las empresas en la frontera hasta un $60 \%$ de las mismas (Tabla 13).

TABLA 13

CLASIFICACIÓN GENÉRICA DEA DE EFICIENCIA EMPRESAS GRUPO 4

\begin{tabular}{|l|c|c|c|c|}
\hline & 2008 & 2009 & 2010 & 2011 \\
\cline { 2 - 5 } & $\%$ & $\%$ & $\%$ & $\%$ \\
\hline $\begin{array}{l}\text { Empresas eficientes. Puntuación de } \\
\text { eficiencia del 100\% }\end{array}$ & $40,00 \%$ & $60,00 \%$ & - & $60,00 \%$ \\
\hline $\begin{array}{l}\text { Empresas marginalmente eficientes. } \\
\text { Puntuación de eficiencia comprendida } \\
\text { entre el 90\% y el 99,99\% }\end{array}$ & - & - & - & - \\
\hline $\begin{array}{l}\text { Puntuación de eficiencia comprendida } \\
\text { entre el 80\% y el 89,99\% }\end{array}$ & & & & - \\
\hline Puntuación de eficiencia inferior al 80\% & $60,00 \%$ & $40,00 \%$ & & \\
\hline
\end{tabular}

Fuente: Elaboración propia

En el período analizado los potenciales de mejora de las empresas consideradas como grandes (véase Tabla 14) se sitúan fundamentalmente en los “otros gastos de explotación” que llegaron a alcanzar un porcentaje del $75 \%$ en el año 2008. En el período 2009 se reparten los potenciales de mejora de forma proporcional en los cuatro factores de producción considerados como inputs en el análisis realizado. Existen empresas caracterizadas como "grandes" que presentan holguras en el importe de la cifra de negocios. 
TABLA 14

POTENCIALES DE MEJORA EMPRESAS GRUPO 4

\begin{tabular}{|c|c|c|c|c|}
\hline & 2008 & 2009 & 2010 & 2011 \\
\hline & $\%$ & $\%$ & $\%$ & $\%$ \\
\hline Aprovisionamientos & - & $20,00 \%$ & - & - \\
\hline Gastos de personal & $25,00 \%$ & $20,00 \%$ & $16,67 \%$ & $33,33 \%$ \\
\hline $\begin{array}{l}\text { Otros gastos de explo- } \\
\text { tación }\end{array}$ & $75,00 \%$ & $20,00 \%$ & $50,00 \%$ & - \\
\hline $\begin{array}{l}\text { Amortización del inmo- } \\
\text { vilizado }\end{array}$ & - & $20,00 \%$ & $16,67 \%$ & $33,33 \%$ \\
\hline $\begin{array}{l}\text { Importe neto de la cifra } \\
\text { de negocios }\end{array}$ & - & $20,00 \%$ & $16,67 \%$ & $33,33 \%$ \\
\hline
\end{tabular}

Fuente: Elaboración propia

\section{CONSIDERACIONES FINALES Y CONCLUSIONES}

La importancia del autobús como servicio de transporte público, la obligada intervención de Administraciones Públicas, en su caso, para garantizar la adecuada ordenación del sector, así como el indudable interés de las empresas y agentes que en él intervienen, entre otras múltiples razones, bien justifican la realización de trabajos del y para el referido sector. En este estudio nos aproximamos a la realidad del sector con una orientación hacia la eficiencia de la prestación del servicio, en municipios de más de 50.000 habitantes, realizando una primera aproximación a los niveles de eficiencia que presentan las empresas que prestan el servicio de transporte urbano de pasajeros por carretera en España, utilizando para ello el análisis envolvente de datos.

De esta primera aproximación a la eficiencia de las empresas analizadas, resaltar que son pocas (13 empresas en 2011), a nuestro juicio, las que presentan la situación ideal, con una eficiencia, tanto global como puramente técnica, igual a 1, sin tener por consiguiente tampoco ineficiencias de escala. No obstante, la categoría de empresas eficientes sin considerar la escala sufre un ligero aumento, mientras que el número de empresas con puntuaciones inferior al $80 \%$ apenas sufre variación (1 empresa).

Dentro de los potenciales de mejora, destaca la partida de gastos de personal en número de holguras, lo cual no es sorprendente ya que si analizamos la estructura de gastos de estas empresas con relación a su cifra de negocios, es la partida más representativa (GP), como puede verse en la Figura 1. 
FIGURA 1

ELEMENTOS DEL GASTO DE LA EXPLOTACIÓN

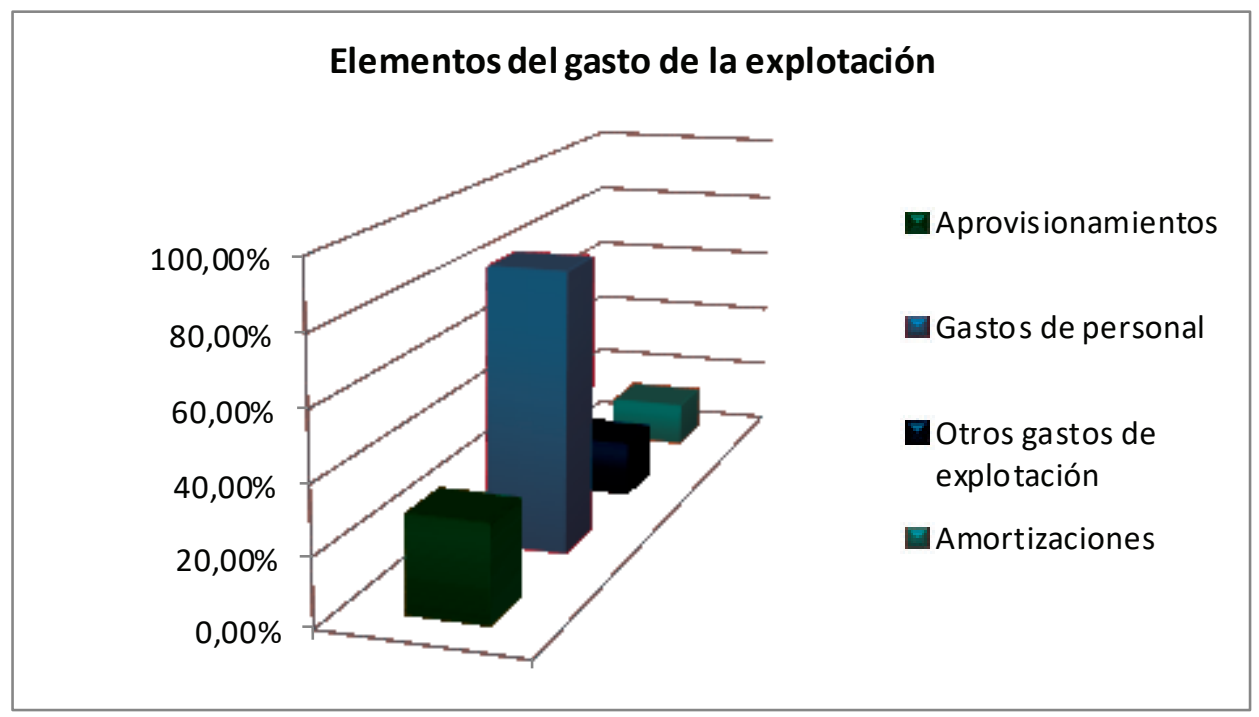

Fuente: Observatorio TUC (2013)

Aunque pudiera pensarse que el tamaño de las empresas es esencial en el logro de una eficiencia puramente técnica, se deduce que se sitúan en la frontera empresas de todos los segmentos considerados, si bien con un mayor número de empresas pequeñas.

Por otra parte, en el período de estudio los potenciales de mejora de las empresas consideradas en cada uno de los segmentos, giran fundamentalmente en torno a los "gastos de personal" y "otros gastos de explotación". No obstante, también se observa una gran dispersión en cada grupo y considerables oscilaciones de un año a otro dentro de los mismos. Resalta como el peso de las holguras en la partida "gastos de personal" es menor en las empresas consideradas "grandes" que en el resto, a excepción de en 2011 que es menor el número de holguras en las de menor dimensión.

No obstante lo anterior, al estar en un sector altamente regulado y dependiente de las subvenciones públicas para la cobertura del déficit del servicio resulta de indudable interés principalmente poder matizar la variable output seleccionada en el trabajo. En este sentido, vemos la necesidad de que las empresas faciliten en su información pública un conjunto significativo de parámetros principalmente de índole técnico que son estrictamente necesarios para que los usuarios de la información puedan hacerse una imagen real y rigurosa de la situación de una empresa y por ende del conjunto del sector.

En esta línea, para mejorar la calidad de las variables a utilizar y la contundencia de las conclusiones a obtener, desde el Observatorio del Transporte Urbano Colectivo se está trabajando para obtener la información técnica, a través del contacto directo con las empresas, y poder contar con algunos parámetros que resultarán claves para realizar análisis comparativo con los resultados de este trabajo.

Es innegable, y así lo exigen los ciudadanos, que un sistema de transporte urbano ha de ser seguro y de calidad, eficiente, equitativo y respetuoso con el medio ambiente, a la vez que debe disponer de recursos financieros para satisfacer sus necesidades de movilidad.

Desde esa perspectiva económica, es esencial analizar los recursos utilizados para la prestación del servicio, de forma que satisfagan en la mayor medida posible, las necesidades humanas, persiguiendo tanto un beneficio social como una eficiencia económica.

Con la financiación que reciben las empresas concesionarias del transporte público urbano por parte de la Administración Pública, debe quedar cubierta la totalidad de los costes de prestación del servicio, una adecuada amortización de su infraestructura y otras prestaciones complementarias.

Sin embargo, asociaciones como ATUC (Asociación de Empresas Gestoras de Transporte Urbano Colectivo) afirman permanentemente que las causas de deterioro de este sector en muchas ciudades deriva de que los fondos financieros recibidos de las Administraciones Públicas son insuficientes, debido fundamentalmente a la crisis 
económica, e inadecuados, motivados por la toma de decisiones apoyadas en criterios más políticos que de gestión.

Desde este punto de vista se considera necesario determinar en qué medida estos fondos son verdaderamente insuficientes o por el contrario, se trata de un problema de ineficiencia en la gestión. Adoptar conclusiones contundentes a este respecto entraña gran dificultad debido a la escasa transparencia informativa de empresas del sector; por un lado, de los aspectos relacionados con la financiación recibida de las Administraciones, y por otro, de las variables técnicas básicas que explican la actividad.

\section{BIBLIOGRAFÍA}

Balboa la Chica, P.M.; Mesa Mendoza, M.; Suárez Falcón, H. (2014): “Análisis de las empresas concesionarias del servicio público de transporte urbano colectivo en España (2008-2010)”. Investigaciones Europeas de Dirección y Economía de la Empresa, Vol. 20 No. 1, 23-32.

Catelló Taliani, E. y Giralt Escobar, S. (2008): “Análisis de la eficiencia en costes de las empresas de transporte de mercancía por carretera: una aproximación empírica del DEA”. Revista Iberoamericana de contabilidad de gestión, nº 11, 93-120.

Coelli, T. (2008): “A guide to DEAP version 2.1: A Data Envelopment Analysis (Computer) Program”. CEPA Working Paper 96/08. Disponible en http:// www.owlnet.rice.edu/ econ380/DEAP.PDF.

De Rus, G., y Herce, J. A. (1996): La regulación de los transportes en España. Civitas.

Farrell, M.J. (1957). "The measurement of productive efficiency". Journal of the Royal Statistical Society, Vol. $120, \mathrm{n}^{\circ} 3$.

Ley 16/1987 de 30 de julio, de Ordenación de los Transportes Terrestres. Boletín Oficial del Estado de 31 de julio de 1987.

Ley 7/1985, de 2 de abril, Reguladora de las Bases del Régimen Local.

López del Pino, F.: Regulación y subvenciones en el transporte público. Una aplicación al caso de Canarias. [Tesis doctoral] Universidad de Las Palmas de Gran Canaria.

Matas, A. y Raymond, J.L. (1998): “Technical characteristics of urban bus companies: The case of Spain”. Trasnportation, 25, 243-263.

Ministerio de Fomento. (2013). Observatorio de Costes del Transporte de Viajeros por carretera. Oferta y demanda. Enero 2013. Secretaría General Técnica. Ministerio de Fomento. Madrid.

Ministerio de Medio Ambiente y Medio Rural y Marítimo y Ministerio de Fomento. Informe del Observatorio de la Movilidad Metropolitana (2009-2011). Observatorio de la Movilidad Metropolitana. 2011. Madrid.

Observatorio de Costes y Financiación del Transporte Urbano Colectivo (OTUC) (2012): Informe Anual del Observatorio TUC 2008-2009. Carrasco Díaz, D. (Coord.) Ed. Instituto de Estudios Económicos (IEE). Madrid.

Observatorio de Costes y Financiación del Transporte Urbano Colectivo (OTUC) (2013): Informe Anual del Observatorio TUC 2008-2011. Carrasco Díaz, D. (Coord.) Ed. Instituto de Estudios Económicos (IEE). Madrid.

Pina, V. y Torres, L. (2001): "Analysis of the efficiency of local government services delivery. An application to urban public transport”. Transportation Research Part A, 35, 929-944.

Real Decreto Legislativo 3/2011, de 14 de noviembre, por el que se aprueba el texto refundido de la Ley de Contratos del Sector Público.

Suárez Falcón, H. (2004): Las Empresas de Transporte de Viajeros por Carretera: Una Propuesta de Modelo Informativo Contable Externo [Tesis Doctoral]. Universidad de Las Palmas de Gran Canaria.

Thomson, J.M. (1976): Teoría económica del transporte, Madrid, Ed. Alianza Universidad. 